12

\title{
Обнаружение малых сигналов в масс-спектрах
}

\author{
(C) Б.А. Калинин, ${ }^{1}$ И.С. Ковалев, ${ }^{1}$ О.С. Тания, ${ }^{1}$ Д.С. Копчук, ${ }^{1,2}$ Г.В. Зырянов, ${ }^{1,2}$ О.Н. Чупахин ${ }^{1,2}$ \\ ${ }^{1}$ Уральский федеральный государственный университет им. Первого президента России Б.Н. Ельцина, \\ 620002 Екатеринбург, Россия \\ ${ }^{2}$ Институт органического синтеза им. И.Я. Постовского УрО РАН, \\ 620219 Екатеринбург, Россия \\ e-mail: kalinin@urfu.ru
}

(Поступило в Редакцию 19 сентября 2016 г.)

\begin{abstract}
Приведены результаты хромато-масс-спектрометрического анализа смеси, содержащей соединение с низкой эффективностью протонирования. Для определения параметров пиков ионов соединения использована программа предварительной обработки масс-спектров. Достоверность результатов обработки подтверждена сравнением с табличными данными масс изотопов и изотопного состава компонента. Проведена расшифровка масс-спектра с вычислением площадей и центроидов пиков с оценкой стандартного отклонения для центра пика. Приведены результаты обработки масс-спектра программой mMass.
\end{abstract}

DOI: $10.21883 / J T F .2017 .09 .44918 .2070$

\section{Введение}

Настоящая работа посвящена обнаружению малых примесей при проведении хромато-масс-спектрометрического анализа. Обнаружение предполагает расшифровку масс-спектра, заключающуюся в идентификации отдельных пиков, на основе измерения отношения массы ионов к заряду и изотопного распределения. Программное обеспечение серийных масс-спектрометров не позволяет в отдельных случаях обнаруживать малые сигналы и не дает возможности анализировать масс-спектры, полученные на другом приборе. В настоящей работе рассмотрено применение программы предварительной обработки масс-спектров для нахождения параметров слабых сигналов, близких к шумовым. Использование программы целесообразно при обнаружении микропримесей и при подавлении части сигналов протонированных молекул. Проведена расшифровка масс-спектра методом вычисления площадей и центроидов пиков с оценкой стандартного отклонения для центра пика.

Приведены результаты обработки масс-спектра программой mMass, написанной Мартином Штрохальмом (Martin Strohalm) (2005-2013 гг.). Последняя имеет открытый исходный код и представлена в Интернете по адресу www.mmass.org.

При взаимодействии 9-(4-бромофенила)-9H-карбазола с mрет-бутиллитием и $\mathrm{CuCl}_{2}$ [1] было получено соединение 1 (рис. 1).

Структура соединения 1 подтверждена спектральными данными ${ }^{1} \mathrm{H}$ и ${ }^{13} \mathrm{C}$ ЯМР спектроскопии, масс-спектрометрии и элементного анализа, которые не противоречат приписываемому строению.

При проведении хромато-масс-спектрометрического анализа реакционной смеси, содержащей химическое соединение 1 с брутто-формулой $\mathrm{C}_{36} \mathrm{H}_{24} \mathrm{~N}_{2}$, при совмещении УФ-хроматограммы (пик $10.9 \mathrm{~min}$ ) указанный компонент в результатах анализа хроматограммы полного ионного тока с использованием программного обеспечения прибора (Compass DataAnalysis 4.0 by Bruker Daltonics) не был обнаружен, напротив, наблюдалось снижение интенсивности ионного тока ниже базовой линии при выходе хроматографического пика вещества 1 (рис. 2).

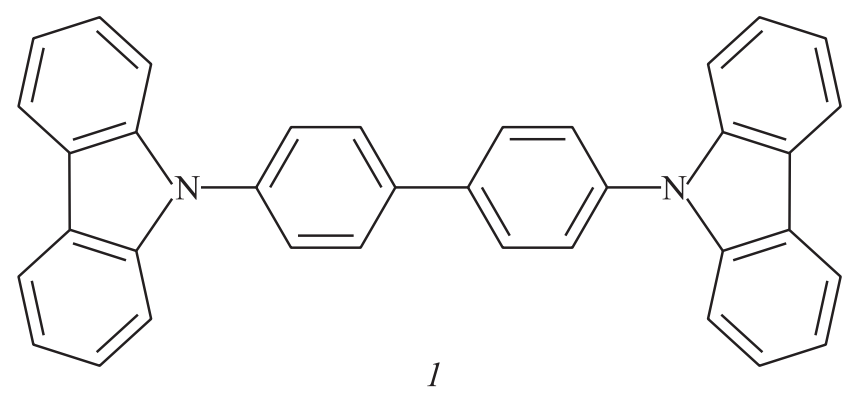

Рис. 1. Соединение 1.

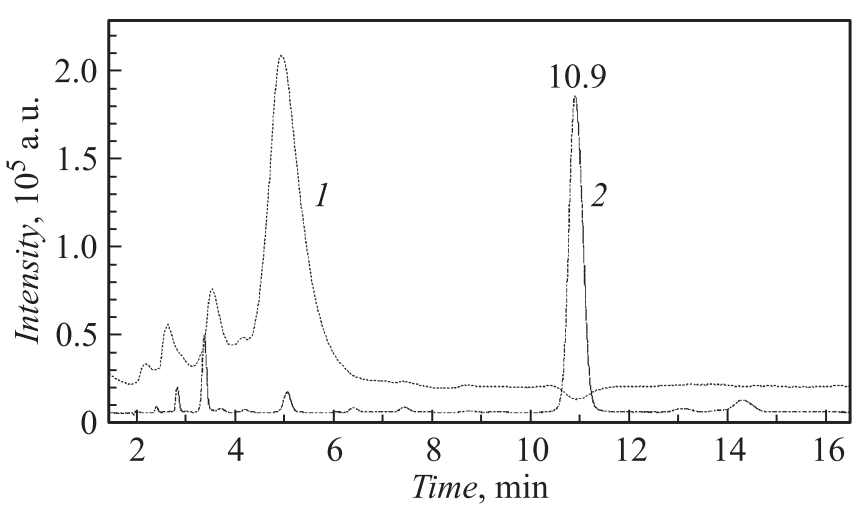

Рис. 2. Хроматограмма реакционной массы, содержащей соединение 1. 1 - хроматограмма полного ионного тока TIC + All MS; 2 -- УФ хроматограмма, $254 \mathrm{~nm}$. 


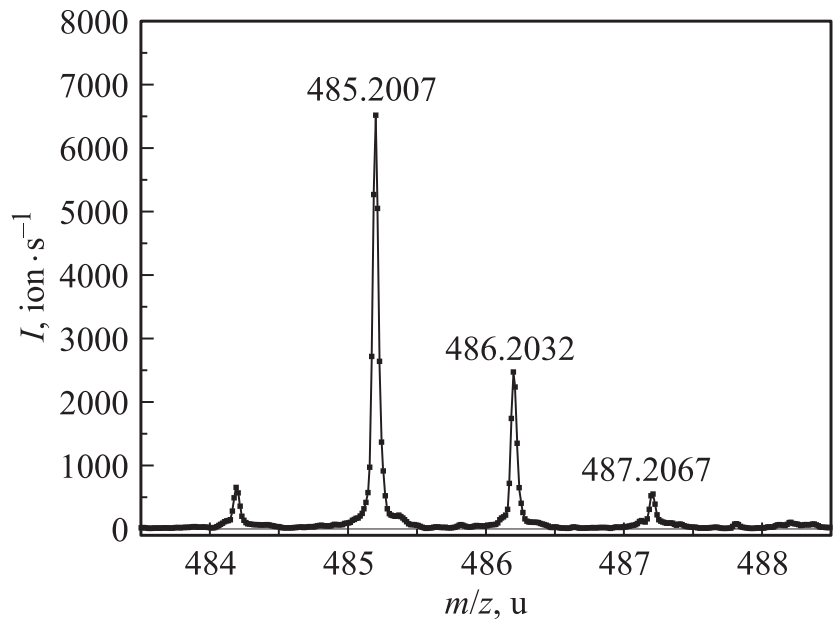

Pис. 3. Участок масс-спектра при прямом вводе анализируемой смеси.

Таблица 1. Параметры расшифровки участка масс-спектра

\begin{tabular}{c|c|c|r|r|c}
\hline$m_{\text {tabl }}, \mathrm{u}$ & $m_{\text {meas }}, \mathrm{u}$ & $B_{\text {meas }}$, ion $/ \mathrm{s}$ & $I_{\text {tabl }}$ & $I_{\text {meas }}$ & $d m, \mathrm{mmu}$ \\
\hline 485.2012 & 485.2007 & 6516 & 66.5 & 68.3 & 0.5 \\
486.2046 & 486.2032 & 2472 & 27.4 & 25.9 & 1.4 \\
487.2079 & 487.2067 & 551 & 5.2 & 5.8 & 1.2
\end{tabular}

В то же время при прямом вводе той же реакционной смеси пики протонированных ионов $\left[\mathrm{C}_{36} \mathrm{H}_{25} \mathrm{~N}_{2}\right]^{+}$присутствуют в масс-спектре (рис. 3).

Параметры расшифровки участка масс-спектра приведены в табл. 1 .

В таблице $m_{\text {tabl }}$ - табличное значение массы иона, $m_{\text {meas }}$ - измеренное значение (из ReportEngine программы Compass), $d m$ - разность табличных и измеренных значений. Последняя не превышает $1.5 \mathrm{mmu}$, что согласуется с нижеприведенной оценкой точности калибровки массовой шкалы. $B_{\text {meas }}$ - измеренная высота интенсивности изотопных массовых пиков. $I_{\text {tabl }}, I_{\text {meas }}-$ табличные и измеренные значения изотопного состава (относительные концентрации) соответственно. Расхождение табличных и измеренных значений изотопных распространенностей не превышает $6 \%$ rel.

\section{Экспериментальная часть}

Масс-спектры высокого разрешения записаны на масс-спектрометре серии micrOTOF-Q II фирмы Bruker Daltonics, оснащенном источником ионизации в электроспрее, шестипортовым краном и устройством прямого ввода kd Scientific (скорость потока $180 \mu \mathrm{l} / \mathrm{h}$ ). Номинальное разрешение прибора 17500 . Реальное разрешение изменялось в ходе записи калибровочного спектра от 5000 до 10000 (для уровня 50\%).

Использован режим регистрации положительно заряженных ионов в диапазоне масс $m / z$ 50-900 u. Внешняя

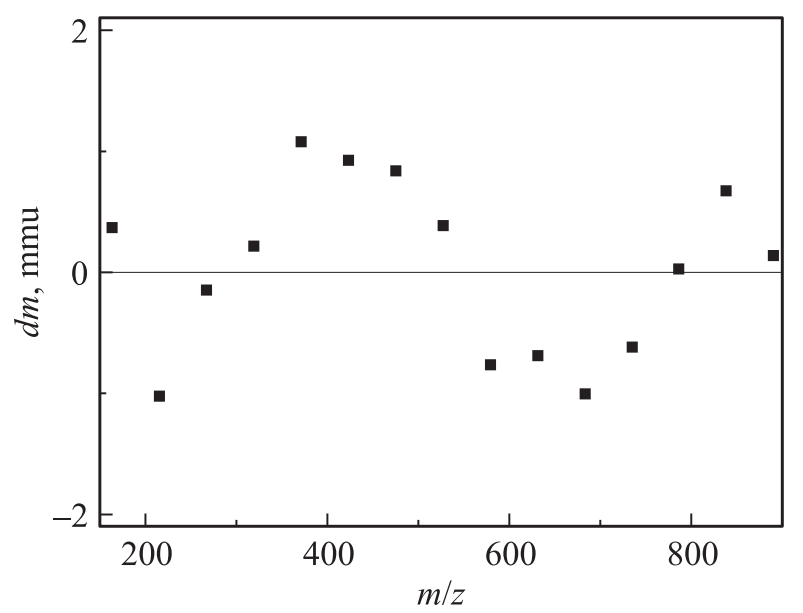

Рис. 4. Калибровочная кривая.

калибровка шкалы масс прибора была проведена по 15 точкам в интервале масс $163-890 \mathrm{u}$. В качестве реперных точек использовались пики кластеров формиата лития при введении в прибор $10 \mathrm{mmol} / 1$ раствора $\mathrm{LiOH}$ в смеси 2-PrOH-0.2\% водного раствора $\mathrm{HCOOH} \mathrm{(1:1}$ по объему). С помощью программы предварительной обработки масс-спектра, описанной ниже, точность калибровки оценена величиной $0.8 \mathrm{mmu}$ (рис. 4).

Сбор и обработка данных выполнялись с использованием программного обеспечения Compass 1.3 for micrOTOF компании Bruker. В качестве хроматографической части использовался хроматограф Agilent 1200 с диодно-матричным УФ-видимым детектором и оснащенный колонкой $4.6 \times 250$ Agilent Zorbax Eclipse XDB C-18. Параметры хроматографического процесса: канал $A-5 \%$ деионизированная вода с добавкой $0.1 \%$ муравьиной кислоты $(5 \%)$, канал $B-95 \%$ ацетонитрил, скорость потока $0.8 \mathrm{ml} / \mathrm{min}$, изократическое элюирование. Детектирование на длине волны $254 \mathrm{~nm}$.

\section{Результаты и обсуждение}

Использование разработанной в нашей лаборатории программы Spectr13 [2] предварительной обработки масс-спектра позволило в автоматическом режиме обнаружить в масс-спектре фрагмента хроматограммы пики с массами 485 и 486 и увидеть вероятное присутствие пика 487 массы (рис. 5).

Программа включает расчет положения, высоты, ширины пиков, уровня фона, а также стандартных отклонений положения и высоты. Суть обработки состоит в автоматическом разбиении всего масс-спектра на зоны пиков и аппроксимации каждого пика функцией Гаусса по методу наименьших квадратов. Масс-спектры для обработки представляются в текстовом формате в виде двух колонок: значения отношений масс к зарядовым числам ионов и интенсивности сигнала детектора. Программа распознает ASCI-II формат ×.xy без дополни- 
Таблица 2. Параметры расшифровки участка масс-спектра (программа Spectr13)

\begin{tabular}{c|c|c|c|c|c|c|c|c}
\hline$m_{\text {tabl }}, \mathrm{u}$ & $m_{\text {mean }}, \mathrm{u}$ & $\Delta m_{\text {mean }}, \mathrm{u}$ & $m_{\text {meas }}, \mathrm{u}$ & $\Delta m_{\text {meas }}, \mathrm{mmu}$ & $B, \mathrm{ion} / \mathrm{s}$ & $I_{\text {tabl }}$ & $I_{\text {meas }}$ & $d m, \mathrm{mmu}$ \\
\hline 485.2012 & 485.1984 & 1.24 & 485.1983 & 0.71 & 251 & 66.5 & 72.7 & 2.9 \\
486.2046 & 486.2010 & 0.99 & 486.2011 & 0.45 & 89 & 27.5 & 25.8 & 3.5 \\
487.2079 & 487.2273 & 13.8 & 487.2249 & 2.9 & 5 & 5.4 & 1.4 & -17
\end{tabular}

тельных преобразований. Результаты расчета параметров пиков переносятся в программу Microsoft Excel для сортировки пиков по ширине и высоте и последующего отбора пиков для калибровки массовой шкалы. Программа апробирована при анализе масс-спектра метастабильных ионов [3] на масс-спектрометре МИ-1201 АГМ и рассмотрении влияния изотопного состава и ненасыщенности связей на спектр дефектов массы остаточных углеводородов [4].

Программа с описанием работы, инструкцией пользователя и масс-спектрами, рассматриваемыми в настоящей работе, представлена в Интернете

http://mp.fizteh.urfu.ru/mass\%20spectrometry.

Основной недостаток программы - невозможность обработки совмещенных пиков. Дополнительно следует отметить неудачную реализацию автоматического разбиения масс-спектра на зоны, размер которых достигает $1 \mathrm{u}$. Такие размеры зон приводят к занижению величины стандартных отклонений параметров кривых аппроксимации в 4-5 раз, по сравнению с выбором (в „ручном режиме“) ширины зоны, равной ширине пика в его основании. Следует, однако, отметить, что расхождение значений самих параметров для этих двух режимов (ручного и автоматического) не превышает величины стандартного отклонения для ручного режима. Так, например, основной параметр 485 пика: положение вершины пика изменяется при смене варианта обработки на $0.012 \mathrm{mmu}$. Это оправдывает применение автоматического режима обработки с оценкой погрешности измерений ручного режима.

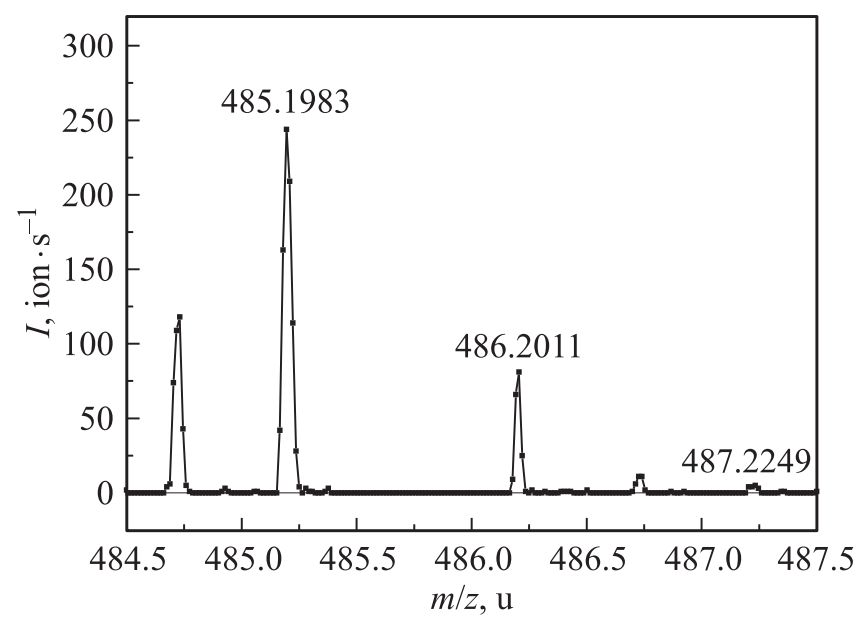

Рис. 5. Участок масс-спектра фрагмента хроматограммы (10.9 min), режим полного ионного тока (8 сканирований).
Таблица 3. Участки масс-спектров для 8 сканирований

\begin{tabular}{c|c|c|c|c|c|c|c|c|c}
\hline$m / z$ & 1 & 2 & 3 & 4 & 5 & 6 & 7 & 8 & Sum \\
\hline 487.1764 & 0 & 0 & 0 & 0 & 0 & 0 & 0 & 0 & 0 \\
487.1905 & 0 & 0 & 0 & 0 & 0 & 0 & 0 & 0 & 0 \\
487.2045 & 0 & 0 & 0 & 0 & 1 & 2 & 1 & 0 & 4 \\
487.2186 & 0 & 0 & 0 & 0 & 1 & 2 & 1 & 0 & 4 \\
487.2327 & 1 & 1 & 0 & 1 & 1 & 1 & 0 & 0 & 5 \\
487.2467 & 1 & 1 & 0 & 1 & 0 & 0 & 0 & 0 & 3 \\
487.2608 & 0 & 0 & 0 & 0 & 0 & 0 & 0 & 0 & 0 \\
487.2749 & 0 & 0 & 0 & 0 & 0 & 0 & 0 & 0 & 0
\end{tabular}

На рис. 5 представлен результат суммирования восьми последовательных сканирований.

Параметры расшифровки участка масс-спектра с помощью программы Spectr13 представлены в табл. 2.

Рассчитанные по отдельным сканированиям значения масс ионов позволяют определить средние значения измеренных масс $\left(m_{\text {mean }}\right)$ и их стандартные отклонения $\left(\Delta m_{\text {mean }}\right)$, рассчитанные по формулам $m_{\text {mean }}=\Sigma m i / 8$; $\Delta m_{\text {mean }}=\left[\Sigma\left(m i-m_{\text {mean }}\right)^{2 / 7}\right]^{1 / 2}$. Исключение составляют ионы с массой $487.2 \mathrm{u}$. Их число в отдельных сканированиях равно в среднем двум (см. табл. 3), что потребовало использования для определения величин $m_{\text {mean }}$ и $\Delta m_{\text {mean }}$ программы Spectr13 в ручном режиме.

Остальные величины в табл. 2 рассчитаны для суммарного масс-спектра (рис. 5): $m_{\text {meas }}$ - измеренное значение массы, $\Delta m_{\text {meas }}$ - стандартное отклонение (рассчитаны из МНК модели программой Spectr13 в ручном режиме), $d m$ - разность табличных и измеренных значений. $B-$ измеренная высота интенсивности изотопных массовых пиков; $I_{\text {tabl }}, I_{\text {meas }}$ - табличные и измеренные значения изотопного состава (относительные концентрации) соответственно.

Параметры расшифровки участка масс-спектра методом вычисления площадей и центроидов пиков с оценкой стандартного отклонения для центра пика [5] представлены в табл. 4.

В качестве меры интенсивности сигнала взята площадь пика $S$. Остальные обозначения те же, что и в табл. 2. Расчет среднего значения массы $m_{\text {mean }}$ для ионов с массой 487.2 в данном случае не вызвал затруднений. При оценке стандартного отклонения для центра пика $\Delta m_{\text {meas }}$ использован расчет среднеквадратичной ширины пика.

Результаты расшифровки участков масс-спектров для обоих методов согласуются с точностью $0.5 \mathrm{mmu}$. Рас- 
Таблица 4. Параметры расшифровки участка масс-спектра (метод вычисления площадей и центроидов пиков)

\begin{tabular}{c|c|c|c|c|c|c|c|c}
\hline$m_{\text {tabl }}, \mathrm{u}$ & $m_{\text {mean }}, \mathrm{u}$ & $\Delta m_{\text {mean }}, \mathrm{u}$ & $m_{\text {meas }}, \mathrm{u}$ & $\Delta m_{\text {meas }}, \mathrm{mmu}$ & $S$, ion $/ \mathrm{s}$ & $I_{\text {tabl }}$ & $I_{\text {meas }}$ & $d m, \mathrm{mmu}$ \\
\hline 485.2012 & 485.1994 & 1.54 & 485.1993 & 0.61 & 804 & 66.5 & 80.2 & 1.9 \\
486.2046 & 486.2013 & 1.49 & 486.2013 & 0.81 & 182 & 27.5 & 18.2 & 3.3 \\
487.2079 & 487.2275 & 13.5 & 487.2248 & 3.7 & 16 & 5.4 & 0.5 & -16.9
\end{tabular}

Таблица 5. Сравнение результатов обработки участка спектра программами Spectr13 и mMass

\begin{tabular}{c|c|r|r|r|r|r}
\hline$m_{1 \text { meas }}, \mathrm{u}$ & $B_{1}$, ion $/ \mathrm{s}$ & $m_{\text {2meas }}, \mathrm{u}$ & $B_{2}$, ion $/ \mathrm{s}$ & \multicolumn{1}{c}{$d m, \mathrm{u}$} & \multicolumn{1}{c|}{$I_{1}$} & \multicolumn{1}{c}{$I_{2}$} \\
\hline 485.1983 & 250.8 & 485.1996 & 230.6 & -1.3 & 72.6 & 74.1 \\
486.2011 & 89.4 & 486.2015 & 76.3 & -0.4 & 25.9 & 24.5 \\
487.2249 & 5.1 & 487.2248 & 4.4 & 0.1 & 1.5 & 1.4
\end{tabular}

хождение с табличными данными для масс 485.2 и $486.2 \mathrm{u}$ не превышает величины $3.5 \mathrm{mmu}$. Табличные и измеренные значения изотопных распространенностей для этих пиков качественно согласуются. Пик ионов с массой $487.2 \mathrm{u}$ имеет демонстрационный характер, не противореча изотопному распределению и, кроме того, показывает преимущества метода вычисления площадей при регистрации предельно малых сигналов.

Участки масс-спектров в диапазоне масс 487.17-487.28 для 8 сканирований и суммарный масс-спектр приведены выше в табл. 3.

Применение программы Spectr13 позволяет обнаруживать пики в автоматическом режиме. При обработке части масс-спектра (прямой напуск) в диапазоне масс 477-490 u программа Spectr13 обнаруживает 27 пиков, тогда как программа Compass приводит данные по 8 пикам. Расхождения в значениях масс для этих пиков не превышают в сравниваемых программах величины $1 \mathrm{mmu}$.

В табл. 5 приведено сравнение результатов обработки участка масс-спектра фрагмента хроматограммы программами Spectr13 и mMass. Цифры в наименованиях величин обозначают, что эти величины были рассчитаны программами Spectr13 (1) и mMass (2).

В табл. $5 m_{1 \text { meas }}, m_{2 \text { meas }}$ - измеренные значения масс ионов, $d m$ - разность измеренных значений; $B_{1}, B_{2}-$ измеренные высоты интенсивности изотопных массовых пиков; $I_{1}, I_{2}$ - измеренные значения изотопного состава.

Измеренные значения масс ионов в пределах погрешности измерений $(1 \mathrm{mmu})$ совпадают. Хорошо согласуются результаты измерения изотопного состава.

\section{Заключение}

Применение программы предварительной обработки масс-спектров Spectr13 позволило зарегистрировать присутствие во фрагменте хроматограммы соединения с низкой эффективностью протонирования. Стандартное программное обеспечение не позволило решить эту задачу. Сравнение возможностей программы Spectr13 и метода обработки масс-спектра по площади пиков обнаружило преимущества последнего при обработке спектров, содержащих в пике менее трех ионов. Результаты применения программ Spectr13 и программы обработки масc-спектров mMass для обработки слабых сигналов согласуются в пределах погрешности измерений.

\section{Список литературы}

[1] Zyryanov G.V., Kovalev I.S., Egorov I.N., Rusinov V.L., Chupakhin O.N. // Chem. Heterocyc. Compd. 2011. Vol. 47. N 5. P. 571-574.

[2] Соломеин А.А., Калинин Б.А., Александров Л.М. // Аналитика и контроль. 2003. Т. 7. № 1. С. 35-39.

[3] Казгов М.А., Калинин Б.А., Александров О.Е., Волобуев П.В. // Масс-спектрометрия. 2009. Т. 8. № 1. С. 15-19; J. Anal. Chem. 2011. Vol. 66. N 14.

[4] Калинин Б.А., Соломеин А.Р., Александров О.Е., Атанов В.Е. // ЖТФ. 2002. Т. 75. Вып. 2. С. 129-130.

[5] Разников В.В., Пихтелев А.Р., Разникова М.О. // Массспектрометрия. 2006. Т. 3. № 2. С. 113-130. 